\title{
Características medias de vórtices cónicos en aristas de cubiertas planas de edificaciones bajas cuboidales utilizando velocimetría por imágenes de partículas
}

\section{Mean Characteristics of Conical Vortices Above Roof Eaves of Low-Rise Cubic Buildings Using Particle Image Velocimetry}

\author{
M. Gamboa-Marrufo \\ Universidad Autónoma de Yucatán, Facultad de Ingeniería. México. \\ E-mail:gmarrufo@uady.mx \\ C.J. Wood \\ Department of Engineering Science, University of Oxford. \\ E-mail:colin.wood@eng.ox.ac \\ R. Belcher \\ Department of Engineering Science, University of Oxford, UK. \\ E-mail:rex.belcher@eng.ox.ac
}

(Recibido: diciembre de 2006; reevaluado: junio de 2008; aceptado: noviembre de 2008)

\section{Resumen}

Los vórtices que se generan cerca de las aristas de techos planos, cuando la dirección del viento forma un ángulo considerable con una esquina del edificio, producen succiones en estas áreas. En flujos turbulentos, los vórtices varían su posición e intensidad y las presiones en la superficie fluctúan consecuentemente. Una primera aproximación para estudiar la relación instantánea entre vórtices y presiones involucra el conocimiento de la posición y características medias de las velocidades del viento en el vórtice. Hasta la fecha, los estudios del flujo se han visto limitados ya que los anemómetros disponibles únicamente miden velocidades en un punto. En este trabajo se utilizó un cubo de $200 \mathrm{~mm}$ para modelar un edificio de $15 \mathrm{~m}$, colocado a $45^{\circ}$ de la dirección del viento y un sistema de velocimetría por imágenes de partículas que permitió capturar vectores de velocidad instantánea de secciones transversales del flujo, tanto en planos transversales como paralelos al eje del vórtice. Estos mapas de vectores se utilizaron para estimar las características medias de los vórtices y recomendar direcciones de planos de observación para utilizar en el estudio de la relación instantánea entre velocidades y presiones.

Descriptores: Velocimetría por imágenes de partículas (PIV), túnel de viento, flujo turbulento, vórtices cónicos en techos, velocidades del viento.

\section{Abstract}

Fluctuating low pressures near the edges of flat roofs are often caused when the wind impinges on one corner of the building so that conical vortices form above the diagonal roof edges. In turbulent flow, these vortices vary in position and strength and the underlying surface pressures fluctuate accordingly. A preliminary approach to the study of the mechanism linking instantaneous roof edge pressures with the wind vortical structures involves the evaluation of mean characteristics and positions of the latter. However the flow 
examination has so far been severely limited by the restriction of available anemometers to single-point sampling. In this experimental study, a $200 \mathrm{~mm}$ cube has been used to model a building with a flat square roof set at an angle of $45^{\circ}$ to the oncoming flow direction, and a Particle Image Velocimetry system was used to capture instantaneous two-dimensional velocity vector images of entire flow cross-sections, both normal to the vortex axis and in planes parallel to that axis. These vector maps were used to estimate the mean characteristics of the vortices and appropriate observation-plane directions to measure wind velocities in the study of the instantaneous problem.

Keywords: Particle Image Velocimetry (PIV), wind tunnel experiments, turbulent flow, roof conical vortices, wind velocity measurements.

\section{Introducción}

Cuando la dirección del viento incidente forma un ángulo considerable con la esquina de un edificio cúbico, se forman dos vórtices tipo ala-delta a lo largo de las aristas del mismo (figura 1). Los vórtices están relacionados con las succiones que se observan en estas áreas y numerosos autores han realizado estudios asociándolos con las altas succiones que se generan en esas áreas. (Marwood, 1996; Pindado and Meseguer, 2003; Melbourne, 1993; Tieleman, 2003). Las succiones son una de las causas principales del daño que se generan en los acabados de las techumbres.

Los estudios de flujo realizados a la fecha se encuentran severamente limitados por la falta de anemómetros capaces de medir simultáneamente direcciones y velocidades del viento en varios puntos de un área. Hasta el momento, muchos esfuerzos se han realizado en aplicar la tecnología que mide velocidades en un solo punto para determinar campos bidimensionales de

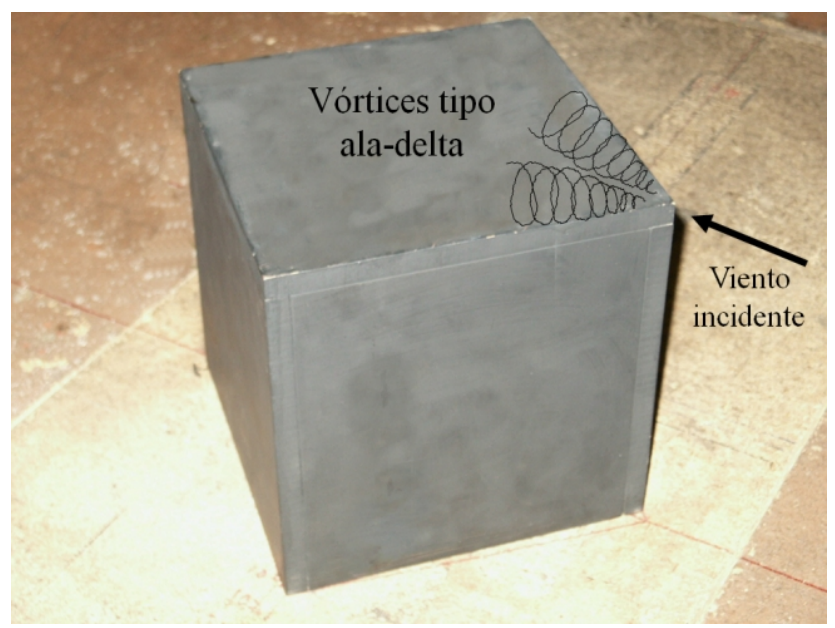

Figura 1. Vórtices en las aristas de edificios cúbicos velocidades de viento; sin embargo, las suposiciones planteadas por los autores para desarrollar estas técnicas introducen incertidumbres al proceso y consecuentemente disminuyen la confiabilidad de sus resultados.

En flujos turbulentos los vórtices varían su posición e intensidad y las presiones en las superficies por debajo de los vórtices fluctúan consecuentemente. Una primera aproximación para estudiar esta relación instantánea involucra el conocimiento de la posición y características medias de los vórtices que se generan en el techo. Este trabajo utilizó un cubo de $200 \mathrm{~mm}$ para modelar un edificio de $15 \mathrm{~m}$, colocado a $45^{\circ}$ de la dirección del viento y presenta la utilización de un sistema de velocimetría por imágenes de partículas que permitió capturar vectores de velocidad instantánea de secciones transversales del flujo, tanto en planos transversales como paralelos al eje del vórtice. Los mapas de vectores se utilizaron para estimar las características medias de los vórtices y recomendar direcciones de planos de observación para utilizar en el estudio de la relación instantánea entre velocidades y presiones. De esta manera, el presente trabajo contribuye a disminuir las incertidumbres de las investigaciones realizadas con anterioridad, en lo que respecta a las mediciones del campo de velocidades del viento y presenta por primera vez las características medias de los vórtices a partir de campos de velocidades bidimensionales más realistas y exactos para ser utilizadas como base para el estudio de los eventos instantáneos que se presentan en la proximidad de las aristas de edificaciones bajas con cubiertas planas.

\section{Sistema coordenado de referencia}

Antes de iniciar cualquier discusión, se requiere establecer una descripción inequívoca de la posición en el flujo arriba del cubo posicionado diagonalmente a la dirección prevaleciente del viento. En este artículo, el origen 
del sistema coordenado principal es la esquina superior a sotavento del cubo. Las posiciones y direcciones desde este punto se describen utilizando un sistema de coordenadas $x, y, z$ de mano derecha, donde $x$ se mide horizontalmente en la dirección contraria a la del flujo incidente y $z$ se mide verticalmente desde la esquina a sotavento al nivel del techo del modelo (figura 2, parte superior). Las componentes de la velocidad en las direcciones de $x, y$ y $z$ son, $u, v$ y $w$, respectivamente.

La parte superior de la figura 2 muestra la localización de los ejes coordenados principales y la numeración de los seis orificios en los que se desea medir la presión en la superficie horizontal del techo del cubo. Los orificios 1, 2 y 3 se encuentran sobre una línea recta, inclinada 10 grados con respecto a la arista del techo. Un sistema de ejes coordenados secundario $x^{\prime}, y^{\prime}, z^{\prime}$ de mano derecha con origen en la esquina superior a sotavento del cubo, se ubicó de tal manera que el eje $x^{\prime}$ pase por los orificios 1, 2 y 3 (figura 2, parte inferior). La dimensión del cubo era de $200 \mathrm{~mm}$ y los orificios 1,2 y 3 se encontraban a 50,100 y $150 \mathrm{~mm}$ de la esquina a sotavento del techo, respectivamente. Las componentes de la velocidad en las direcciones de $x^{\prime}, y^{\prime}$ y $z^{\prime}$ son, $u^{\prime}, v^{\prime}$ y $w^{\prime}$, respectivamente.

De la misma manera, los orificios 4,5 y 6 se encuentran sobre una línea recta, inclinada 15 grados con respecto a la arista del cubo, también a 50,100 y $150 \mathrm{~mm}$ de la esquina a sotavento. Otro sistema de ejes coordenados secundario $x^{\prime \prime}, y^{\prime \prime}, z^{\prime \prime}$ de mano derecha con origen en la esquina superior a sotavento del cubo se ubicó de tal manera que el eje $x^{\prime \prime}$ pase por los orificios 4,5 y 6

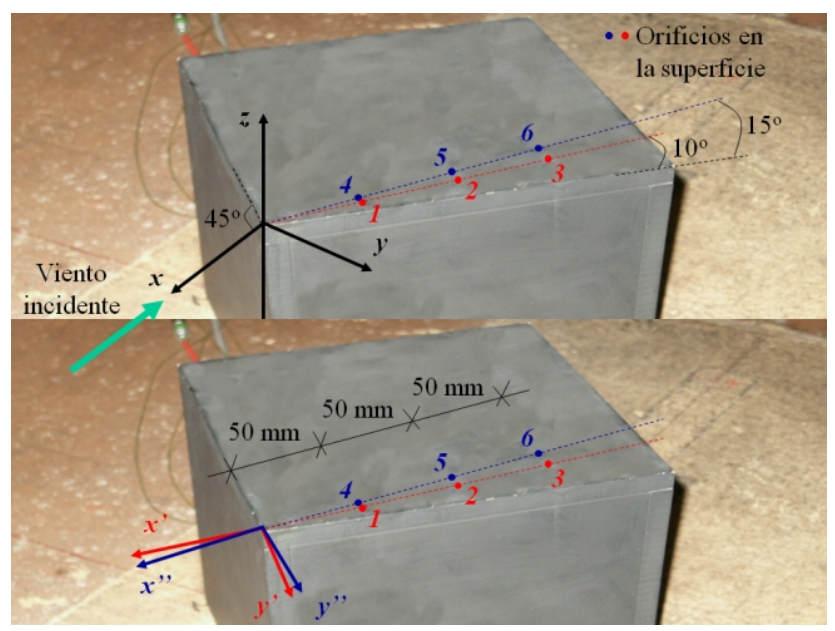

Figura 2. Parte superior: Sistema de ejes coordenados principal $(x, y, z)$ y orificios para medir presiones en la superficie del techo. Parte inferior: Sistemas de ejes coordenados secundarios $\left(x^{\prime}, y^{\prime}, x^{\prime \prime}, y^{\prime \prime}\right)$ (figura 2, parte inferior). Las componentes de la velocidad en las direcciones de $x^{\prime \prime}, y^{\prime \prime} y z^{\prime \prime}$ son, $u^{\prime \prime}, v^{\prime \prime} y w^{\prime \prime}$, respectivamente. Las líneas a 10 y 15 grados de los seis orificios para medir presiones se escogieron de tal manera que los orificios quedaran debajo de las posiciones más probables del centro del vórtice medio de acuerdo con una revisión bibliográfica previa (Gamboa- Marrufo, 2004).

\section{Simulación de la capa límite}

Los experimentos descritos en este trabajo se realizaron en el túnel de viento ambiental de $4 \mathrm{~m} \times 2 \mathrm{~m}$ del Departamento de Ingeniería de la Universidad de Oxford. Se simuló una capa límite atmosférica utilizando una rejilla al inicio del área de trabajo (sitio de entrada del flujo) de $15 \mathrm{~m}$ del túnel de viento y una distribución de elementos que simularan la rugosidad a lo largo de este último. La modelación de la capa límite se realizó igualando las características de los perfiles de velocidad media y de intensidad de la turbulencia, así como de los espectros de turbulencia longitudinal en el túnel de viento con aquellos representativos de una capa límite atmosférica real, obtenidos de las correlaciones de información a escala real publicadas en las Unidades de Información de Ciencias de la Ingeniería (ESDU) británicas. (ESDU, 1995a; ESDU, 1995b; ESDU, 1995c; y ESDU, 1995d). El objetivo fue modelar, a escala 1:75, la capa límite de viento que puede esperarse sobre un terreno con construcciones tipo suburbano. Para esta capa límite, las ESDU sugieren un valor de $0.25 \mathrm{~m}$ para la intersección $z_{0}$ en el perfil de velocidades logarítmico definido por:

$$
\ln z_{0}=\ln z-\frac{\bar{u}_{z}}{2.5 u, K_{x}}
$$

Donde, $\bar{u}_{z}$ es la velocidad media a una altura $z$ sobre el nivel del suelo, $u *$ es la velocidad de fricción y $K x$ es el factor de rugosidad local que considera los cambios en la rugosidad de la superficie en dirección contraria al viento incidente.

Las figuras 3, 4 y 5 muestran la comparación entre las características del flujo en el túnel de viento y la información a escala real de las ESDU. Los parámetros comparados son los perfiles de velocidad media, de intensidad de la turbulencia y el espectro de turbulencia longitudinal a una altura de $15 \mathrm{~m}$ a escala real, respectivamente. 

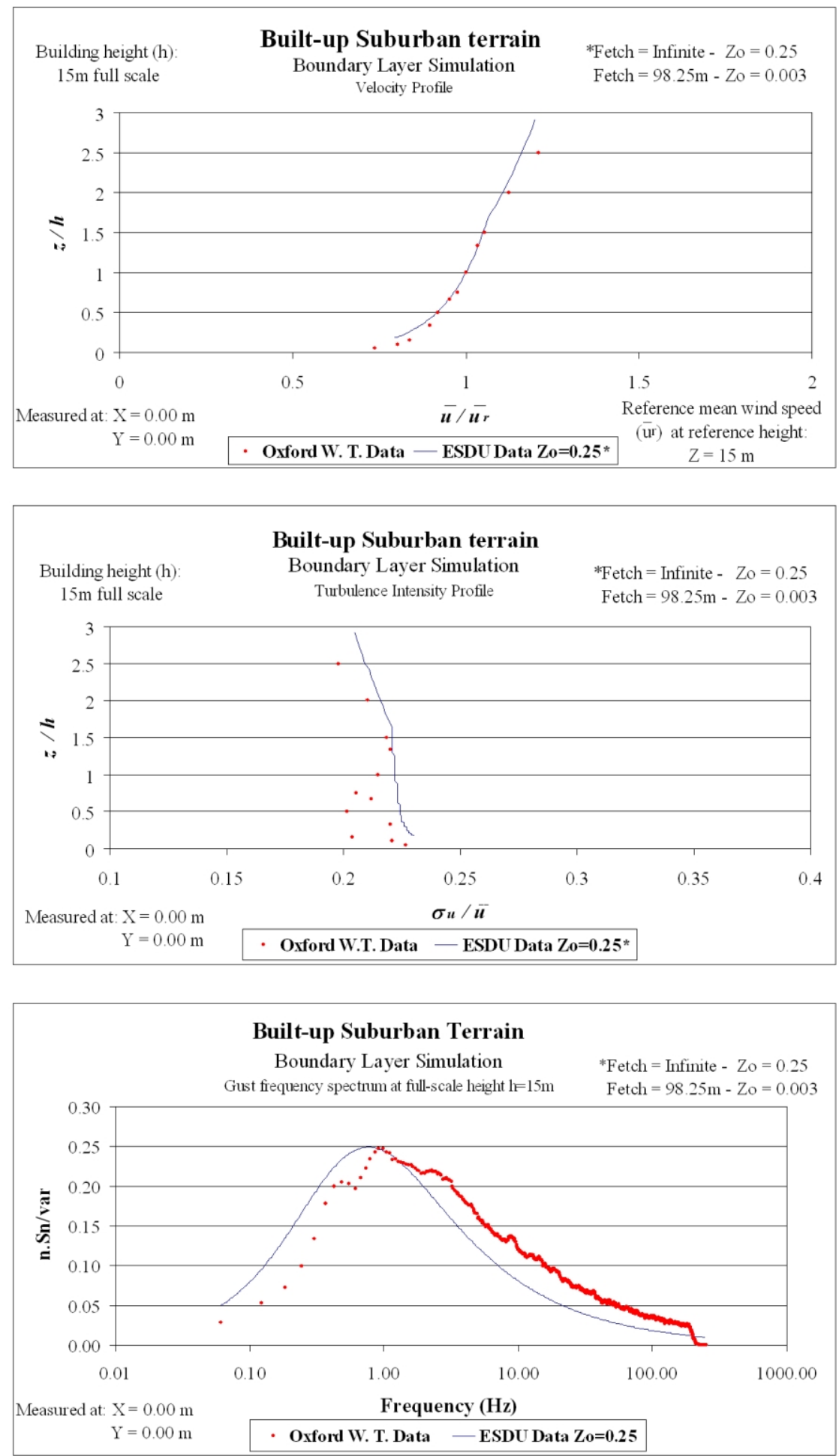

Figuras 3, 4 y 5. Simulación de la capa limite de un terreno con construcciones suburbanas. Perfiles de velocidad media, de intensidad de la turbulencia y espectros de frecuencia de ráfagas en el túnel de viento de la Universidad de Oxford e información a escala real de ESDU, $z_{0}=0.25 \mathrm{~m}$. 
DOI: http://dx.doi.org/10.22201/fi.25940732e.2009.10n2.011

M. Gamboa-Marrufo, C.J. Wood, R. Belcher

\section{Modelación a escala}

Con el fin de obtener similitud dinámica entre los eventos en el flujo modelado y el atmosférico a escala real, los experimentos se deben modelar a través del análisis dimensional para que todos los parámetros adimensionales correspondientes tengan los mismos valores en el modelo y a escala real. Una descripción más detallada de la similitud dinámica la dan Massey, 1998; Cook, 1985a y Cook, 1985 b.

Para los experimentos contenidos en este trabajo, las variables que se cree influyen en los mismos se presentan con valores típicos en la tabla 1. La tabla 2 contiene los parámetros adimensionales correspondientes y muestra la extensión a la cual estos parámetros fueron igualados durante los experimentos en el túnel de viento.

Sin embargo, definir parámetros adimensionales e igualar requerimientos es una cosa y alcanzar tal igualación durante la experimentación práctica es otra. En particular, en ingeniería eólica es prácticamente imposible igualar el número de Reynolds modelado y el de la atmósfera a escala real.

Los valores presentados están referidos a un modelo de edificio a escala $1: 75$, sujeto a una velocidad nominal del túnel de viento de $4.5 \mathrm{~m} / \mathrm{s}$. El prototipo correspondiente es un edificio cúbico de $15 \mathrm{~m}$ de altura. La igualdad en la frecuencia adimensional (tabla 2) determina toda la escala del tiempo entre el modelo y el prototipo. Así, por ejemplo, a una velocidad de viento de $13 \mathrm{~m} / \mathrm{s}$ a escala real, los 90 segundos de información de PIV (ver Obtención de datos por medio de PIV) representan 39 minutos de flujo de viento del prototipo elegido, mientras que la frecuencia de obtención de las imágenes de $15 \mathrm{~Hz}$ del PIV (ver Obtención de datos por medio de PIV) corresponden a $0.577 \mathrm{~Hz}$ a la escala del prototipo.

\section{Medición de velocidades}

Las mediciones de velocidades se realizaron con la diagonal del techo alineada con la dirección del viento

Tabla 1. Lista de parámetros y valores típicos

\begin{tabular}{cccc}
\hline Nombre de la variable & Símbolo & Valor a escala real & Valor en el modelo \\
\hline $\begin{array}{c}\text { Descripción del edificio } \\
\text { Descripción del viento }\end{array}$ & $\mathrm{h}$ & 15 & 0.2 \\
Altura del edificio y nominal del viento de referencia $(\mathrm{m})$ & & & \\
Velocidad media de referencia del viento a $15 \mathrm{~m}(\mathrm{~m} / \mathrm{s})$ & $\bar{u}_{r}$ & 13 & 4.5 \\
Perfil de velocidades medias $(\mathrm{m} / \mathrm{s})$ & $\bar{u}$ & Fig. 3 & Fig. 3 \\
Perfil de desviaciones estándar de ráfagas $(\mathrm{m} / \mathrm{s})$ & $\sigma_{u}$ & Fig. 4 & Fig. 4 \\
Frecuencia de ráfagas a la altura de referencia $(\mathrm{Hz})$ & $\mathrm{n}$ & Fig. 5 & 1.225 \\
Densidad nominal del aire $\left(\mathrm{Kg} / \mathrm{m}^{3}\right)$ & $\rho$ & 1.225 & $1.8 \times 10^{-5}$ \\
Viscosidad nominal del aire $(\mathrm{Kg} / \mathrm{ms})$ & $\mu$ & $1.8 \times 10^{-5}$ & Fig.
\end{tabular}

Tabla 2. Lista de parámetros adimensionales. Los valores en el modelo y a escala real deben ser iguales

\begin{tabular}{cccc}
\hline Nombre de variables & Símbolo & Valor a escala real & Valor en el modelo \\
\hline Descrpción del viento & & & \\
Razón de la velocidad media a una altura determinada & $\bar{u} / \bar{u}_{r}$ & Fig. 3 & Fig. 3 \\
Intensidad de la turbulencia a una altura determinada & $\sigma_{u} / \bar{u}$ & Fig. 4 & Fig. 4 \\
Frecuencia de ráfagas a la altura de referencia & $n h / \bar{u}$ & Fig. 5 & Fig. 5 \\
Número de Reynolds nominal & $\rho \overline{u_{r}} h / \mu$ & $1.3 \times 10^{7}$ & $6.1 \times 10^{4}$ \\
\hline
\end{tabular}


incidente, de tal manera que las aristas del techo formaran un ángulo de $45^{\circ}$ con esta última. Los planos de observación del flujo fueron todos planos verticales localizados de modo que incluyeran a la línea vertical que pasa por el orificio para medir presiones. Rotando el plano de iluminación y la cámara sobre esta línea vertical, los planos de observación pueden posicionarse a cualquier ángulo para obtener las componentes seleccionadas de la velocidad del viento local. Los planos verticales básicos fueron paralelos y normales a las líneas a $10^{\circ}$ y $15^{\circ}$ de la arista del techo.

\section{Obtención de datos por medio de PIV}

La Velocimetría por Imágenes de Partículas (PIV) consiste en adquirir pares de imágenes de partículas en movimiento en el flujo. Para lograr lo anterior, un plano del flujo se ilumina con una lámina de luz láser emitida en pulsaciones y una cámara fotográfica se coloca para visualizar este plano perpendicularmente. La cámara registra las posiciones de las partículas en dos fotos, utilizando pulsaciones de luz consecutivas con un intervalo de tiempo conocido entre ellas y a partir de esta información se obtienen los vectores de velocidad en el plano del flujo iluminado.

El equipo utilizado para medir las velocidades del viento en el presente trabajo fue un sistema de PIV suministrado por Dantec Measurement Technology (DMT) que, junto con los generadores de partículas, mecanismos de sincronización y otros equipos existentes en el laboratorio del grupo de investigación en ingeniería eólica de la Universidad de Oxford (OU-WERG), permitieron realizar las cinco tareas requeridas para la adquisición de datos utilizando PIV, específicamente, generación de partículas, iluminación, registro, procesamiento y análisis de campos de flujo (Dantec, 2000).

La generación de partículas se realizó utilizando una mezcla 5:1 de agua/glicerina a través de dos atomizadores modelo 9306 Six-Jet de TSI, Inc. y, en ocasiones en que se requirió mayor densidad de partículas, se utilizó un generador de humo basado en aceite y manufacturado por CF Taylor, Ltd. Las partículas generadas en el fluido fueron iluminadas utilizando un sistema láser Nd:YAG modelo Gemini-15Hz de NewWave Research, Inc. Este equipo permite iluminar un plano del flujo con dos pulsaciones de luz láser un máximo de 15 veces por segundo; el intervalo entre las dos pulsaciones se controla por un disparador externo y puede ser tan corto como $5 \mu \mathrm{s}$.

El registro de imágenes fue realizado por una unidad de adquisición y control con procesador modelo
FlowMap PIV 1100 de DMT, a través de una cámara fotográfica modelo 80C42 DoubleImage 700 de DMT, utilizando una lente de $28 \mathrm{~mm}$ o de $60 \mathrm{~mm}$, según requerido por el área de visualización y distancia a la que se colocó la cámara para no afectar al flujo en estudio. La cámara contiene un chip CCD de 768x484 pixeles, electrónica especializada para el almacenamiento por separado de dos imágenes con intervalos entre ellas que van de $2 \mu$ s a 66 ms y una frecuencia máxima entre captura de pares de imágenes de $30 \mathrm{~Hz}$. Así, el chip CCD se expone al reflejo de la luz láser en las partículas en el plano del flujo iluminado por la primera pulsación del láser, se limpia y se expone al reflejo de luz láser debido a la segunda pulsación; posteriormente, ambas imágenes se transfieren al procesador FlowMap PIV 1100.

El procesamiento y análisis de los campos de flujo se realizaron con la información almacenada en el procesador durante la adquisición de imágenes y los softwares incluidos en éste y en una PC utilizada para controlar a todo el sistema (FlowMap Basic y Flow Manager Analysis, respectivamente). En cada experimento se adquirieron 1351 pares de imágenes (90 segundos de información del flujo). El intervalo de tiempo entre imágenes de un par varió entre 10 y $25 \mu$ s y la frecuencia entre pares de imágenes fue de $15 \mathrm{~Hz}$ (67 ms).

Cada imagen estaba registrada en una matriz de $768 \times 484$ pixeles, que fue dividida en sub-áreas de 64x64 pixeles superpuestas un $50 \%$ en ambas direcciones, horizontal y vertical, para calcular el desplazamiento espacial promedio de las partículas en el intervalo de tiempo entre imágenes de un par de ellas por medio de una correlación cruzada en cada sub-área. Cada imagen quedó dividida en un arreglo de 23×14 sub-áreas (322 sub-áreas por imagen).

Se utilizó una función de ventana Gaussiana con el parámetro $k=1.19$ para mejorar la tasa entre la señal y el ruido cíclico generado por las Transformaciones de Fourier realizadas durante la correlación cruzada, y un filtro Gaussiano atenuador de altos (low-pass) con un parámetro $k=3$ ó 4 para ampliar los picos de correlación estrechos en el dominio de las frecuencias. La función de ventana Gaussiana, implementada como filtro de entrada al algoritmo de la correlación cruzada, determinó un ancho de ventana de 54 pixeles (85\% del ancho de la sub-área) y como filtro de salida del mismo, el filtro Gaussiano atenuador de altos, aseguró anchos de picos de correlación de 3 a 6 pixeles, valores que garantizan estar utilizando toda la información válida contenida en cada sub-área de las imágenes.

Una vez que se determinó el vector de desplazamiento $d$ en pixeles para cada sub-área, se calculó el 
DOI: http://dx.doi.org/10.22201/fi.25940732e.2009.10n2.011

M. Gamboa-Marrufo, C.J. Wood, R. Belcher

correspondiente grupo de vectores de velocidad utilizando la ecuación:

$$
v=S \frac{d}{\tau}
$$

Donde, $\tau$ es el intervalo entre imágenes de un par y $S$ es el factor de escala entre el objeto y su representación en la imagen. Este último fue variable en cada experimento dependiendo de la distancia focal del lente de la cámara y la distancia entre la posición de la cámara y el área de mediciones.

Como validación de los mapas de vectores obtenidos en cada experimento (1351 mapas con 322 vectores por mapa), en el presente trabajo se utilizó una razón entre señal y ruido de 1.2, un rango de 3 a 6 pixeles para el ancho de los picos de correlación y un rango de velocidades aceptables de $\pm 15 \mathrm{~m} / \mathrm{s}$ para asegurar la remoción de vectores ilegítimos y retener aquéllos en los extremos del rango de velocidades medidas.

Una descripción más detallada de la técnica de velocimetría por imágenes de partículas se proporciona en la Guía de Usuarios e Instalación, FlowMap Instrumentación de Velocimetría por Imágenes de Partículas (PIV) de Dantec (Dantec, 2000).

\section{Resultados y discusión}

\section{Posición y descripción del vórtice medio}

Con el propósito de determinar la localización del centro del vórtice medio y direcciones aproximadas de las velocidades medias en las secciones inferior y superior de éste a diferentes distancias de la esquina a sotavento del cubo, se observó el flujo encima de los orificios para medir presiones en planos paralelos y perpendiculares a las líneas formadas por los mismos a 10 y 15 grados, con respecto a la arista del cubo (direcciones paralelas a los ejes $x^{\prime}, y^{\prime}, x^{\prime \prime}$ e $y^{\prime \prime}$ en la figura 2). Las figuras 6 y 7 muestran los campos de velocidades medias sobre los orificios para medir presiones 2 y 5 , respectivamente sobre los ejes $x^{\prime} y x^{\prime \prime}$. Se obtuvieron patrones de variación de velocidades similares sobre los orificios 1, 3, 4 y 6 . En estas figuras los ejes $x^{\prime} y x^{\prime \prime}$ tienen el origen en la esquina a sotavento del plano del techo del modelo (figura 2). Las figuras 8 y 9 presentan las velocidades medias también encima de los orificios para medir presiones 2 y 5 , sobre ejes paralelos a $y^{\prime}$ e $y^{\prime \prime}$. Las coordenadas auxiliares paralelas a $y^{\prime}$ e $y^{\prime \prime}$ tienen el origen en el orificio para medir presiones ( 2 y 5 , respectivamente). Se obtuvieron patrones de variación de velocidades similares sobre los orificios $1,3,4$ y 6 .

Las figuras 6 a 9 describen apropiadamente a los vórtices obtenidos con velocidades medias sobre las cubiertas de edificios. El diagrama de flujo en la figura 6 muestra que el plano de observación de las velocidades sobre el eje $x^{\prime}$ corta al vórtice medio en el lado en el que las velocidades tienen dirección hacia arriba, mientras que el diagrama de flujo de la figura 7 muestra el plano sobre el eje $x^{\prime \prime}$ en el que las velocidades tienen dirección hacia abajo. Esto es consistente con las velocidades medias mostradas en las figuras 8 y 9 que ilustran que el centro del vórtice medio se encuentra entre $11.5^{\circ}$ y $13.3^{\circ}$ de la arista del edificio (la variación en los ángulos obtenidos se debe a las diferentes perspectivas en las que se miden las velocidades en los planos perpendiculares a los ejes $x^{\prime \prime}$ y $x^{\prime}$ en $x^{\prime \prime} / h=-0.25$ y $x^{\prime} / h=-0.25$, respectivamente); entre $11.75^{\circ}$ y $12.2^{\circ}$ (cuando las velocidades son medidas en $x^{\prime \prime} / h=-0.5 y x^{\prime} / h=-0.5$, respectivamente) y entre
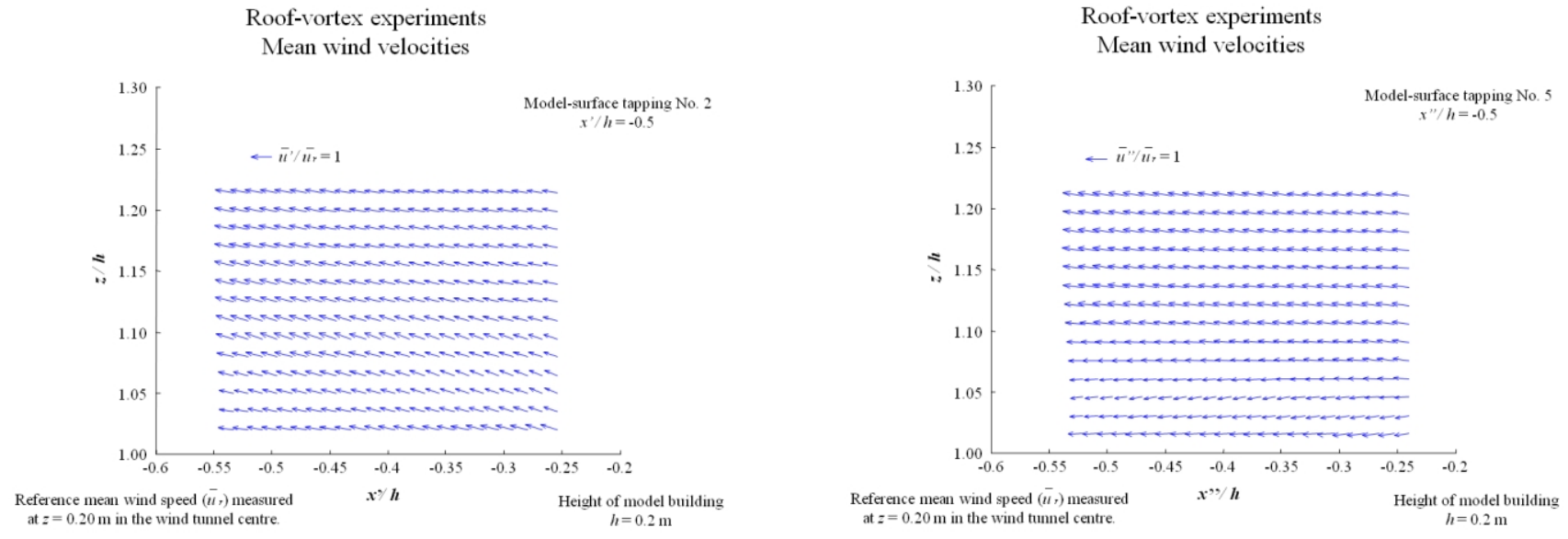

Figura 6 y 7. Velocidades medias sobre los orificios para medir presiones Num. 2 (sobre el eje x') y Num. 5 (sobre el eje x") 
$12.1^{\circ}$ y $12.6^{\circ}$ (cuando las velocidades se miden en $x^{\prime \prime} / h=-0.75 y x^{\prime} / h=-0.75$, respectivamente).

Las figuras 8 y 9 también muestran que el centro del vórtice medio forma un ángulo vertical de aproximadamente $4.13^{\circ}$ con el plano del techo del edificio (altura del centro del vórtice. Por lo tanto, el vórtice medio crece uniformemente con la distancia a la esquina a sotavento del edificio.

El flujo se pega nuevamente a la superficie del techo en un punto localizado entre $\mathrm{y}^{\prime} / \mathrm{d}=0.20 \mathrm{y} \mathrm{y} / \mathrm{d}=0.25 \mathrm{o}$ entre $\mathrm{y}^{\prime \prime} / \mathrm{d}=0.15 \mathrm{y} \mathrm{y}^{\prime \prime} / \mathrm{d}=0.20$ (una vez más, la obtención de un rango en estos valores se debe a las perspectivas de los planos de medición de las velocidades).

Se realizaron dos experimentos por cada plano de observación con excepción del orificio activo No. 6 en el que se realizaron tres experimentos con el plano de medición sobre el eje $y^{\prime}$. Todos los experimentos realizados con un mismo plano de observación proporcionaron las mismas posiciones del centro del vórtice medio.

La tabla 3 presenta las magnitudes de las velocidades medias en puntos seleccionados abajo y arriba del centro de los vórtices medios obtenidos en todos los experimentos realizados. La información presentada en ésta muestra que las velocidades sobre los orificios para medir presiones Nos. 1 a 3 son respectivamente mayores que aquéllas sobre los orificios Nos. 4 a 6.

Esta tabla cuantifica la disminución de las magnitudes de las velocidades del vórtice medio con el aumento de la distancia entre el punto de observación y la esquina a sotavento del edificio, lo cual concuerda con lo reportado por otros autores según una revisión bibliográfica del tema (Gamboa-Marrufo, 2004).

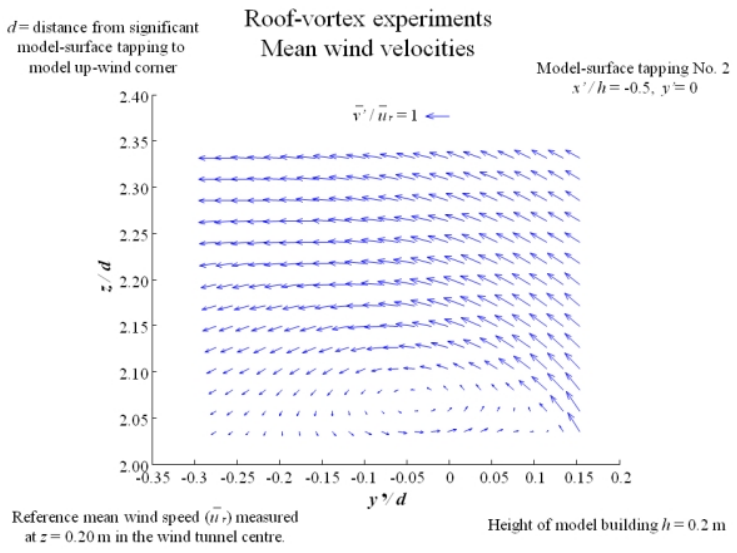

\section{Conclusiones}

Posición y descripción del vórtice medio

Los mapas de vectores obtenidos con velocimetría por imágenes de partículas se utilizaron por primera vez para estimar las características medias de los vórtices que se generan cerca de las aristas de techos planos, cuando la dirección del viento forma un ángulo de 45 grados con una arista del edificio, y recomendar direcciones de planos de observación para medir velocidades en el estudio de la relación instantánea entre velocidades del viento y las presiones generadas en las cercanías de las aristas de techos planos.

Se obtuvieron las direcciones y magnitudes correspondientes a los vectores de velocidad media en la parte superior e inferior del vórtice cónico medio en seis puntos de estudio colocados a tres distancias de la esquina de sotavento $(50,100$ y $150 \mathrm{~mm}$ ) y en líneas que forman dos ángulos diferentes (10 y 15 grados) con la arista del techo. Las direcciones resultantes de estas velocidades, calculadas en la tabla 3 , se presentan en la figura $10 \mathrm{co}-$ mo las direcciones de los planos de observación más adecuados propuestos para el estudio de la relación entre las velocidades del viento en los vórtices y las presiones instantáneas generadas en los techos sujetos a un flujo turbulento.

Ya que en las zonas cercanas a las aristas de las cubiertas planas de edificaciones cuboidales se presentan vórtices cónicos (figura 1), las direcciones recomendadas para intentar correlacionar las velocidades instantáneas del viento en la parte superior e inferior del vórtice con las presiones correspondientes varían como se ilustra en la figura 10.

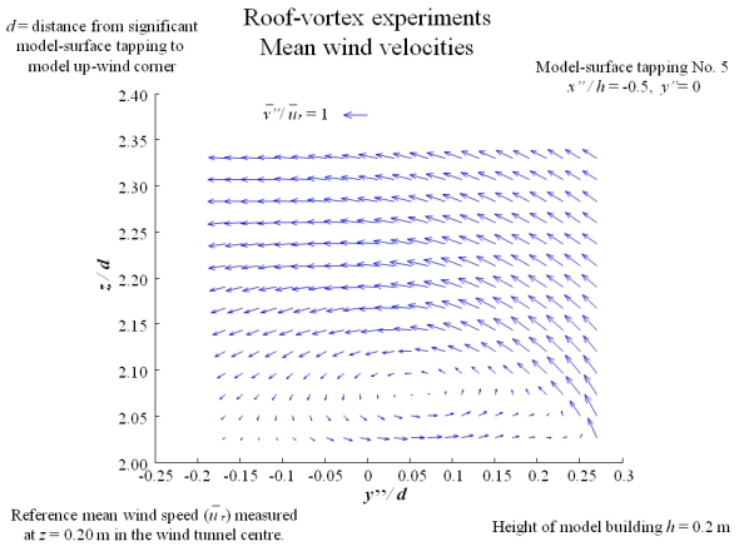

Figuras 8 y 9. Velocidades medias sobre el orificio para medir presiones Num. 2 (sobre el eje paralelo a $y^{\prime}$ ) y Num. 5 (sobre el eje paralelo $y^{\prime \prime}$ ) 
Tabla 3. Velocidades medias en puntos seleccionados abajo y arriba del centro del vórtice medio, obtenidos en los experimentos. Las direcciones resultantes de estas velocidades se ilustran en la figura 10

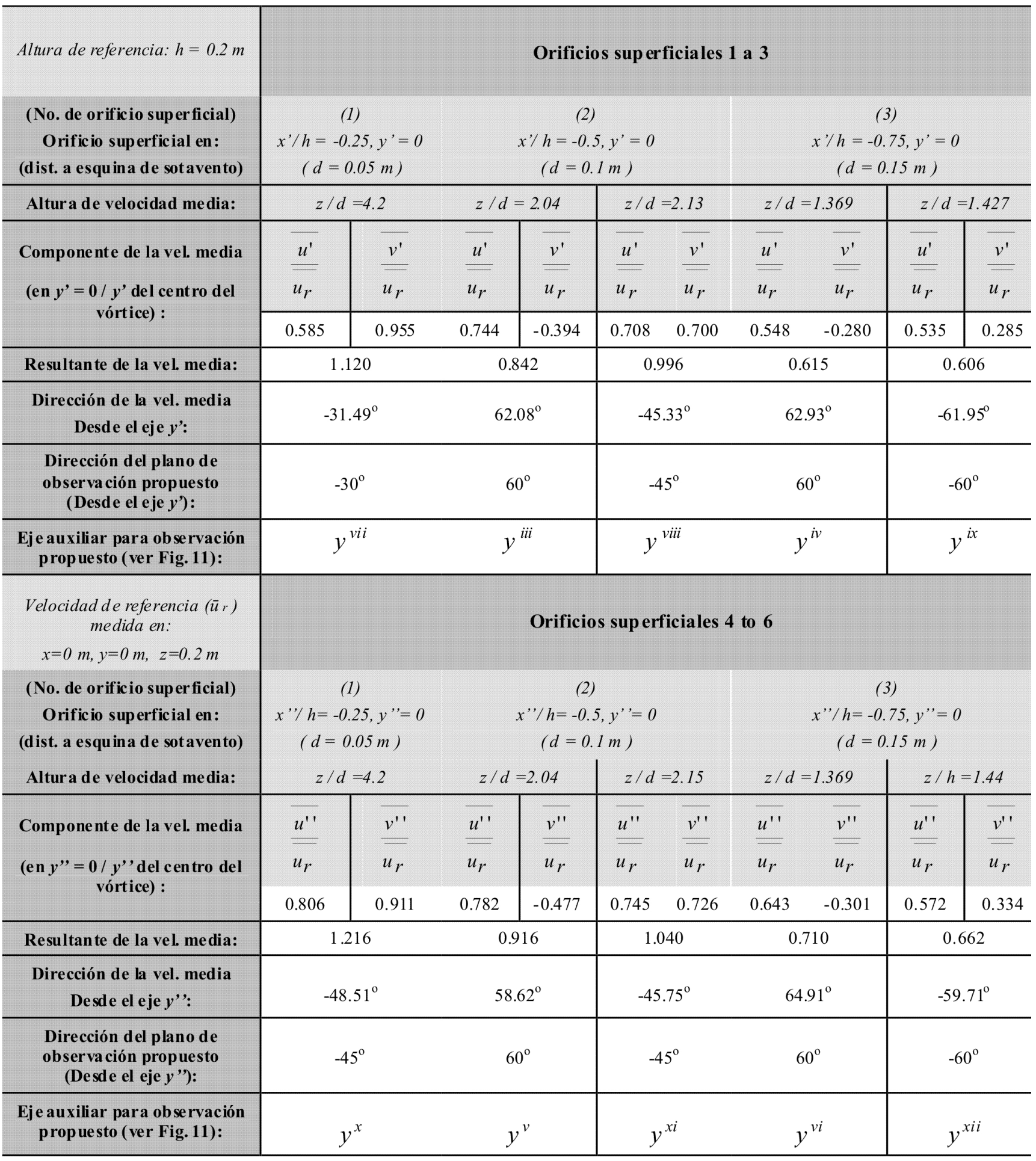


DOI: http://dx.doi.org/10.22201/fi.25940732e.2009.10n2.011

Características medias de vórtices cónicos en aristas de cubiertas planas de edificaciones bajas cuboidales utilizando velocimetría por imágenes de partículas
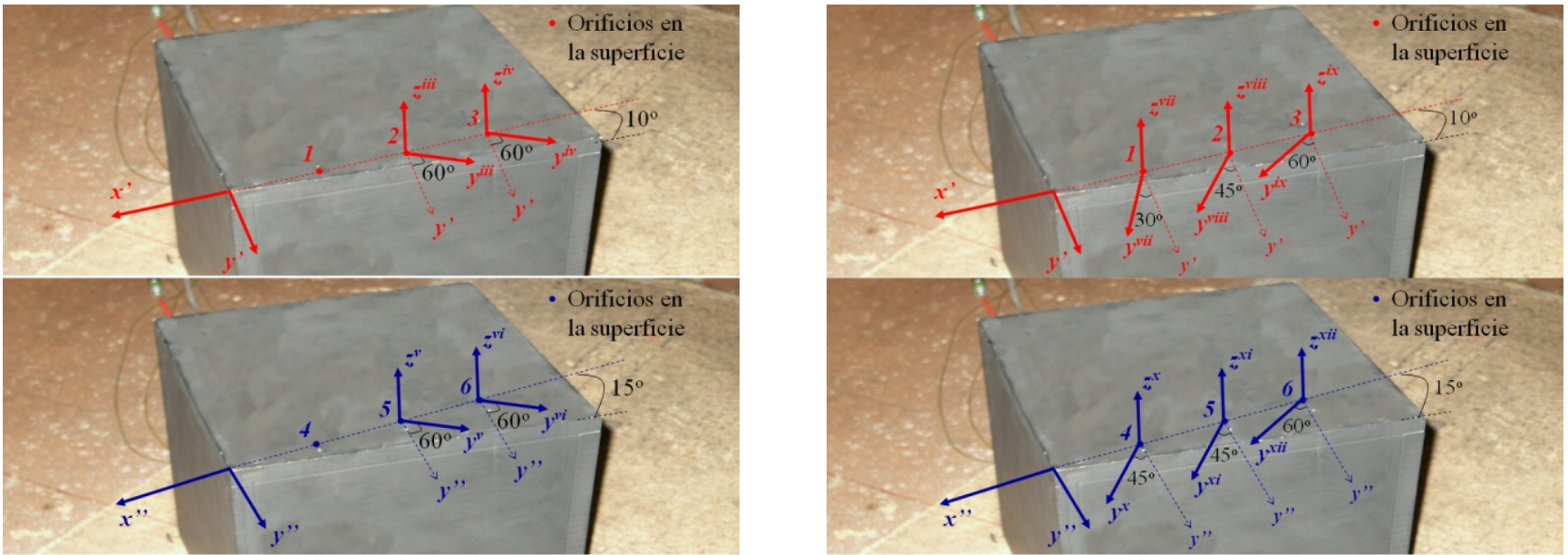

Figura 10. Direcciones recomendadas para el estudio de las velocidades en la parte inferior (izquierda) y superior (derecha) del vórtice

\section{Referencias}

Cook N.J. The Designer's Guide to Wind Loading of Building Structures. Part 1: Background, Damage Survey, Wind Data and Structural Classification. Building Research Establishment Report. Building Research Establishment. Department of the Environment. Butterworths. Great Britain. 1985a.

Cook N.J. The Designer's Guide to Wind Loading of Building Structures. Part 2: Static structures. Building Research Establishment Report. Building ResearchEstablishment. Department of the Environment. Butterworths. Great Britain. $1985 b$.

Dantec. Flow Map Particle Image Velocimetry Instrumentation. Installation and User's Guide. Fifth Edition. Dantec Measurement Technology. Skovlunde, Denmark. Aug. 2000.

ESDU Engineering Sciences Data. Wind Engineering. Strong Winds in the Atmospheric Boundary Layer, Part I: Mean Hourly Wind Speeds, Engineering Science Data Unit Item 82026. 1982 (Amendments April 1993) Issue 29. March, 1995a.

ESDU. Engineering Sciences Data. Wind Engineering. Strong Winds in the Atmospheric Boundary Layer, Part I: Discrete Gust Speeds, Engineering Science Data Unit Item 83045. 1983 (Amendments April 1993). Issue 29. March, $1995 b$

ESDU. Engineering Sciences Data. Wind Engineering. Wind Speed Profiles Over Terrain with Roughness Changes, Engineering Science Data Unit Item 84011.1984 (Amendments April 1993). Issue 13. March 1995c.

ESDU. Engineering Sciences Data. Wind Engineering. Characteristics of Atmospheric Turbulence Near the Ground, Part II: Single Point Data for Strong Winds (Neutral
Atmosphere), Engineering Science Data Unit Item 85020. 1985 (Amendments April 1993). Issue 29. March 1995d.

Gamboa-Marrufo M. Wind Engineering Applications of Particle Image Velocimetry (PIV). Thesis (Submitted in partial fulfilment of the requirements of the degree of Doctor of Philosophy). University of Oxford. Trinity Term, 2004.

Marwood R. An Investigation of Conical Roof Edge Vortices. Thesis (Submitted in partial fulfilment of the requirements of the degree of Doctor of Philosophy). University of Oxford. Trinity Term, 1996.

Massey B. Mechanics of Fluids. Seventh Edition. Revised by John Ward-Smith. Stanley Thornes (Publishers) Ltd. United Kingdom. 1998.

Melbourne W.H. Turbulence and the Leading Edge Phenomenon. Journal of Wind Engineering and Industrial Aerodynamics, 49: 45-64. 1993. Elsevier Science, Amsterdam, The Netherlands.

Pindado S. and Meseguer J. Wind Tunnel Study on the Influence of Different Parapets on the Roof Pressure Distribution of Low-Rise Buildings. Journal of Wind Engineering and Industrial Aerodynamics, 91:1133-1139. 2003. Elsevier Science, Amsterdam, The Netherlands.

Tielman H.W. Wind Tunnel Simulation of Wind Loading on Low-Rise Structures: A Review. Journal of Wind Engineering and Industrial Aerodynamics. Vol. 91:1627-1649. 2003. Elsevier Science, Amsterdam, The Netherlands. 
DOI: http://dx.doi.org/10.22201/fi.25940732e.2009.10n2.011

M. Gamboa-Marrufo, C.J. Wood, R. Belcher

\section{Semblanza de los autores}

Colin John Wood. Doctor en filosofía por el Imperial Collage del Reino Unido. Fundador y director del Grupo de Investigación en Ingeniería de Viento de la Universidad de Oxford desde 1971, diseñó el túnel de viento ambiental de esta institución que comenzó a operar en 1974. Desde entonces y hasta el año 2003, trabajó continuamente realizando consultorías para diversas industrias del Reino Unido e investigación relacionada con la ingeniería eólica desde el Departamento de Ciencias de la Ingeniería de la Universidad de Oxford. Ha dirigido numerosas tesis para esta institución y escrito innumerables artículos y reportes de investigación.

Mauricio Gamboa-Marrufo. Doctor en filosofía por la Universidad de Oxford, Reino Unido, obtuvo la maestría en estructuras del Instituto Técnico de la Universidad Técnica de Lisboa, Portugal, la especialidad en informática en el Instituto Tecnológico de Mérida y la licenciatura en ingeniería civil en la Facultad de Ingeniería de la Universidad Autónoma de Yucatán (FIUADY). Pertenece al Cuerpo Académico de Estructuras y Materiales de la FIUADY desde 1991. En el 2004, inició la investigación en ingeniería eólica en la FIUADY como investigador responsable del proyecto del túnel de viento para la FIAUDY, actualmente en funcionamiento. Fue director y editor fundador de la revista actual de esa Facultad y es miembro del Consejo Editorial de la misma.

Rex Belcher. Técnico del Departamento de Ciencias de la Ingeniería de la Universidad de Oxford y miembro del Grupo de Investigación en Ingeniería de Viento de la misma institución. Se encargó del túnel de viento ambiental del Laboratorio Osney de la Universidad de Oxford desde su inauguración en 1974 hasta el año 2003, en que el equipo fue entregado al Grupo de Investigación en Ingeniería de Turbinas. Ha participado, junto con el Dr. Colin J. Wood, en incontables consultorías para diversas industrias del Reino Unido y proyectos de investigación relacionados con la ingeniería eólica. 